\section{Finnish public health colorectal cancer screening program justifies continuation}

Colorectal cancer screening using the fecal occult blood test (FOBT) reduces colorectalcancer-related mortality; however, the effectiveness of public-health screening programs based on this test remains unproven. Adequate sensitivity of a Finnish, public, colorectal cancer screening program based on the FOBT has now been demonstrated, and this program warrants continuation.

The program, which was initiated in 2004, was designed to enable analysis of its effectiveness. Individuals aged 60-66 years from volunteering municipalities ( $n=106,000)$ were randomly allocated to either a screening or a control group, and FOBT kits were posted to those in the screening group. The returned kits were analyzed at a national center and participants with positive results were advised to contact their local health center for colonoscopy.

Sensitivity of the screen was evaluated in terms of the ability of the FOBT to identify disease (test sensitivity), the combined ability of the FOBT and colonoscopy to identify disease (episode sensitivity), and the ability of the program to identify cancers in the target population (program sensitivity). After a mean follow-up of 1.9 years, the estimated test sensitivity was $54.6 \%$ and episode sensitivity was $51.3 \%$. Although the participation rate was $70.8 \%$, the program sensitivity was $37.5 \%$, which suggests that the program identified over one-third of colorectal cancers in the total population.

Original article Malila N et al. (2008) Test, episode, and programme sensitivities of screening for colorectal cancer as a public health policy in Finland: experimental design. BMJ [doi:10.1136/bmj.a2261]

\section{Fecal calprotectin: a useful diagnostic marker for acute, bacterial diarrhea}

Acute, bacterial diarrhea can be severe and, in some cases, life-threatening. The standard method with which to diagnose acute, infectious diarrhea is bacteriological culture; however, this technique is time-consuming and expensive. Rapid, inexpensive screening methods are, therefore, needed. A study by
Shastri et al. has demonstrated the accuracy of using fecal calprotectin to diagnose acute, bacterial diarrhea.

The study compared the diagnostic accuracy of three tests: fecal calprotectin, fecal lactoferrin and the guaiac-based fecal occult blood test (FOBT). Stool samples from 2,185 patients with acute diarrhea were analyzed by performing cultures for the identification of different enteral pathogens. Samples from patients with a positive microbial diagnosis $(n=195)$ and controls with a negative microbial diagnosis $(n=196)$ were then subjected to the three different tests. The sensitivity and specificity of the fecal calprotectin test were $82.6 \%$ and $87.2 \%$, respectively, compared with $77.9 \%$ and $53.6 \%$ for the fecal lactoferrin test and $37.9 \%$ and $84.7 \%$ for the FOBT. Of note, results from the fecal calprotectin test could be generated within hours.

The superior correlation of the calprotectin test findings with bacteriological, infectious diarrhea suggests that calprotectin is a suitable screening marker for bacterial diarrhea. Stool samples that test positive for calprotectin should then be cultured to provide a definitive diagnosis. Use of this technique should reduce associated costs and, more importantly, the time taken to achieve diagnosis, which leads to improved prognosis for patients.

\section{Original article Shastri YM et al. (2008) Prospective multicenter study evaluating fecal calprotectin in adult acute bacterial diarrhea. Am J Med 121: 1099-1106 \\ Improved diagnostic accuracy of wireless esophageal pH monitoring}

The results of a study by Wenner and colleagues suggest that positioning a $\mathrm{pH}$ electrode directly above the squamocolumnar junction (SCJ) during wireless monitoring of esophageal $\mathrm{pH}$ improves the diagnostic accuracy of the test compared with standard positioning $(6 \mathrm{~cm}$ above the SCJ).

This European, prospective study included patients with typical reflux symptoms that recurred more than twice weekly for the 6 months preceding enrollment. In total, 62 patients ( 39 men and 23 women) were recruited, along with 55 healthy volunteers who acted as controls. Study participants underwent $48 \mathrm{~h}$ wireless $\mathrm{pH}$ monitoring. Two $\mathrm{pH}$ recording 